\title{
(2) OPEN ACCESS \\ Prognostic value of estimated plasma volume in patients with chronic systolic heart failure
}

\author{
Yuyao Lin (1) , 1,2,3 Yanbo Xue, 1,2,3 Jing Liu, 1,2,3 Xiqiang Wang, ${ }^{1,2,3}$ Linyan Wei, ${ }^{1,2,3}$ \\ Ling Bai, ${ }^{1,2,3}$ Aiqun Ma (D) 1,2,3
}

- Additional material is published online only. To view, please visit the journal online (http://dx.doi.org/10. 1136/jim-2020-001538).

\section{Department of}

Cardiovascular Medicine, First Affiliated Hospital of Xi'an Jiaotong University, Xi'an, Shaanxi, China

${ }^{2}$ Key Laboratory of Molecular Cardiology, Ministry of Education, Xi'an, Shaanxi, China

${ }^{3}$ Key Laboratory of Environment and Genes Related to Diseases (Xi'an Jiaotong University), Ministry of Education, Xi'an, People's Republic of China

Correspondence to Dr Aiqun Ma, Xi'an, China; aiqun.ma@xjtu.edu.cn Dr Ling Bai;

bailingb21@sina.com

Accepted 20 October 2020 Published Online First 22 December 2020

\section{ABSTRACT}

Assessing congestion is challenging but important to patients with chronic heart failure (CHF). However, there are limited data regarding the association between estimated plasma volume status (ePVS) determined using hemoglobin/hematocrit data and outcomes in patients with stable CHF. We prospectively analyzed 231 patients; the median follow-up period was 35.6 months. We calculated ePVS at admission using the Duarte and Strauss formula, derived from hemoglobin and hematocrit ratios and divided patients into three groups. The primary outcome was a composite of all-cause mortality or heart failure rehospitalization. Among 274 patients (61.98 years of age, $2.3 \%$ male), the mean ePVS was $3.98 \pm 0.90 \mathrm{dL} / \mathrm{g}$. The third ePVS tertile had a higher proportion of primary outcome $(71.4 \%)$ than the first or second tertile $(48.1 \%$ and $59.7 \%$, respectively; $p=0.013)$. On multivariable Cox analysis, after adjusting for potential confounders, higher ePVS remained significantly associated with increased rate of primary outcome (adjusted HR 1.567, 95\% Cl 1.267 to $1.936 ; p<0.001)$. Kaplan-Meier survival analyses showed that the occurrence of primary outcome, all-cause mortality and rehospitalization increased progressively from first to third tertiles $(p=0.006,0.014$ and 0.001 ; respectively). In receiver operating characteristic analysis, the area under the curve of ePVS for primary outcome was 0.645 . ePVS determined using hemoglobin and hematocrit was independently associated with clinical outcomes for patients with stable CHF. Our study thus further strengthens the evidence that ePVS has important prognostic value in patients with stable CHF.

Trial registration number ChiCTRONC-14004463.

\section{INTRODUCTION}

Chronic heart failure (CHF) is the ultimate pathological outcome of most organic heart disease. ${ }^{1}$ Congestion is a major cause of worsening CHF. ${ }^{2-4}$ Higher degrees of congestion are associated with higher hospitalization rates and adverse outcomes. ${ }^{56}$ Relief of congestion is the basic goal of therapy for patients with $\mathrm{CHF}^{7}$

Congestion is difficult to quantify in a noninvasive way, and therefore it is difficult to measure accurately. ${ }^{3}{ }^{4}$ Currently, there are limited means to quantify plasma volume (PV)

\section{Significance of this study}

What is already known about this subject?

- Congestion is a major cause of worsening chronic heart failure (CHF). Higher degrees of congestion are associated with higher hospitalization rates and adverse outcomes.

- Estimated plasma volume status (ePVS) derived from the Duarte formula was reported to be a simple and effective clinical examination to measure congestion.

- Limited data regarding the association between ePVS determined using hemoglobin/hematocrit data and outcomes in patients with stable CHF.

\section{What are the new findings?}

- ePVS calculated simply from hemoglobin and hematocrit independently provided a predictive value for long-term heart failure hospitalization or mortality outcome in patients with stable systolic CHF.

- ePVS was a better predictor than hemoglobin and hematocrit in the multivariable analysis.

How might these results change the focus of research or clinical practice?

- Hemoglobin and hematocrit to estimate plasma volume (PV) is a low-cost, easily measurable alternative method available in clinical practice. The ePVS measurements may help physicians estimate PV and adjust guideline-based medications on clinical practice in the future.

in patients with CHF. Symptoms and signs can only be used when congestion is apparent. ${ }^{8}$ The respective role of congestion scores in routine clinical practice still remains to be determined. ${ }^{9}$ Lung ultrasound can capture rapid changes in congestion and may represent the extension of clinical examination in patients. ${ }^{9}$ Echocardiographic parameters such as mitral valve E/e', systolic pulmonary artery pressure and inferior vena cava have some limitations, such as the effect of respiratory status of the patients, need of echocardiography training and presence of different of echocardiographers. ${ }^{10} 11$ The radiotracer dilution method is clinically impractical 
and expensive. ${ }^{12}$ Daily weight has little effect over longer periods and can only be used when the patient's 'dry weight' is known. ${ }^{13}$ Additional objective measures of congestion consistent with simple and effective clinical examinations may be helpful. ${ }^{14} 15$

It has been shown that estimated PV status (ePVS) which is calculated from weight and hematocrit, was associated with prognosis in CHF cohorts. ${ }^{15}$ Nevertheless, the dry weight is difficult to measure and agreement between the calculated and measured PV levels in this cohort was appraised only in male patients with CHF. ${ }^{13}{ }^{15}$ ePVS derived from hemoglobin and hematocrit may represent a better tool to reflect congestion. ePVS estimated from the Duarte formula was reported to be associated with clinical outcomes in patients with heart failure with preserved ejection fraction (EF). ${ }^{16} 17$ Nevertheless, despite the published data on the ePVS estimated from the Duarte formula in patients with $\mathrm{CHF},{ }^{18}$ the contribution of ePVS to the clinical outcomes of patients with stable systolic CHF has not been sufficiently investigated. Moreover, as patients with CHF spend most of their time self-managing outside the hospital, ${ }^{19}$ simple and reliable PV monitoring is more meaningful for patients with stable CHF rather than hospitalized patients with $\mathrm{CHF}^{15}$ Therefore, we investigated ePVS using hemoglobin and hematocrit and outcomes in patients with stable systolic CHF in the present study.

\section{MATERIAL AND METHODS}

\section{Study populations}

This was a prospective cohort study. We recruited patients diagnosed with systolic heart failure (HF) according to the '2014 China Heart Failure Diagnosis and Treatment Guideline' and '2012 ESC Guidelines for the Diagnosis and Treatment of Acute and Chronic Heart Failure ${ }^{, 20}$ in Xunyi Hospital and Jingyang Hospital in Shaanxi, China, before and treated in the outpatient clinics from 2014 to 2015. Inclusion criteria were as follows: age 18-80 years; echocardiography showing left ventricular ejection fraction (LVEF) of $<50 \%$; not hospitalized in the past month. All patients were included after the stabilization of both clinical status and medications. According to the guidelines, patients were administered standardized drug treatment regimens ${ }^{20}$ to reduce deviations in patient treatment regimens arising from different medical units and physicians. The ePVS data in this prospective study were not used for treatment decisions. Hypertension was defined as systolic blood pressure (BP) of $\geq 140 \mathrm{~mm} \mathrm{Hg}$, diastolic BP of $\geq 90 \mathrm{~mm} \mathrm{Hg}$, or use of at least one class of antihypertensive agents. ${ }^{21} \mathrm{We}$ defined non-insulin-dependent diabetes mellitus as fasting blood glucose concentration of $>126 \mathrm{mg} / \mathrm{dL}$ and/or use of at least one oral hypoglycemic agent. ${ }^{22}$ Atrial fibrillation was defined according to '2014 AHA/ACC/HRS Guideline for the Management of Patients with Atrial Fibrillation'. ${ }^{23}$ Anemia was defined according to the $\mathrm{WHO}$ criteria as a baseline hemoglobin of $<130 \mathrm{~g} / \mathrm{L}$ for men and $<120 \mathrm{~g} / \mathrm{L}$ for women.

The exclusion criteria were as follows: CHF related to congenital heart diseases, valvular disease or pericardial disease, acute HF, chronic obstructive pulmonary disease or life-threatening malignancy, pregnancy and lactation, and dementia or mental disorders. Note that patients with clinically significant bleeding events were excluded because either transfusion therapy or blood loss would change hemoglobin level, thereby affecting the calculation of ePVS.

\section{Clinical measurement}

We recorded physical and clinical characteristics, medical histories, blood chemistry data and medications at admission. Body mass index (BMI) was determined according to weight in kilogram divided by the square of height in meter $\left(\mathrm{kg} / \mathrm{m}^{2}\right) \cdot{ }^{24}$ Estimated glomerular filtration rate was assessed using the Modification of Diet in Renal Disease 4 variable formula. ${ }^{25}$ We calculated the Meta-analysis Global Group in Chronic Heart Failure (MAGGIC) scores of all subjects to fit the multivariable Cox regression model. ${ }^{26}$

Estimated plasma volume status (ePVS) in this study was calculated from the Strauss-derived Duarte formula using hemoglobin and hematocrit values as follows ${ }^{14}$ : ePVS $=(100-$ hematocrit $(\%)) /$ hemoglobin $(\mathrm{g} / \mathrm{L})$.

\section{Outcomes and follow-up}

The primary outcome was a composite of all-cause mortality or rehospitalization due to worsening HF. The median follow-up period was 35.6 months, and the longest follow-up period was 43 months. Recording study endpoint information was documented via telephone interviews, by regular outpatient follow-up, or by electronic hospital records.

\section{Statistical analysis}

The Kolmogorov-Smirnov test was used to test normal distribution of continuous variables. Normally distributed variables are expressed as mean $\pm \mathrm{SD}$, and median value (25th-75th IQR) for non-normally distributed continuous variables. The frequency of categorical variables is expressed as numbers (n) (percentages (\%)). As the distribution of $\mathrm{N}$-terminal pro-brain natriuretic peptide (NT-proBNP) levels had a skewed distribution, it was normalized with logarithmic transformation (abbreviated as $\log \mathrm{BNP}$ ). The comparisons between ePVS tertile groups showing normal distribution were made using the Student $t$-test or otherwise by the Mann-Whitney U test. We used the Pearson $\chi^{2}$ test to compare categorical data. Correlational statistics (Pearson's rho) between ePVS, $\operatorname{logBNP}$, and LVEF were computed. Correlations of $\mathrm{r} / \mathrm{rho}=0.25$ or greater (absolute value) were noted and $\mathrm{p}$ values were reported. Cox regression analyses were performed to evaluate the associations between ePVS and outcomes. Univariable analyses were used for most baseline variables to find variables that might be associated with primary outcome. Next, all variables that showed a significant $(p<0.10)$ univariate association and which were known to be significant for predicting primary outcome in patients with HF were included in a multivariable model. The multivariate analysis included age, gender, systolic BP, MAGGIC score, logBNP, ePVS, hemoglobin, hematocrit and LVEF. We used 'forward selection' to ensure that variables that did not retain significance $(p>0.10)$ in this multivariable analyses were not added to the final model. Moreover, any variable highly correlated with another variable and with a less significant $\mathrm{p}$ value was not retained. Cox analyses were used to evaluate the survival, while the Harrel concordance index (C-index) was used to testify 


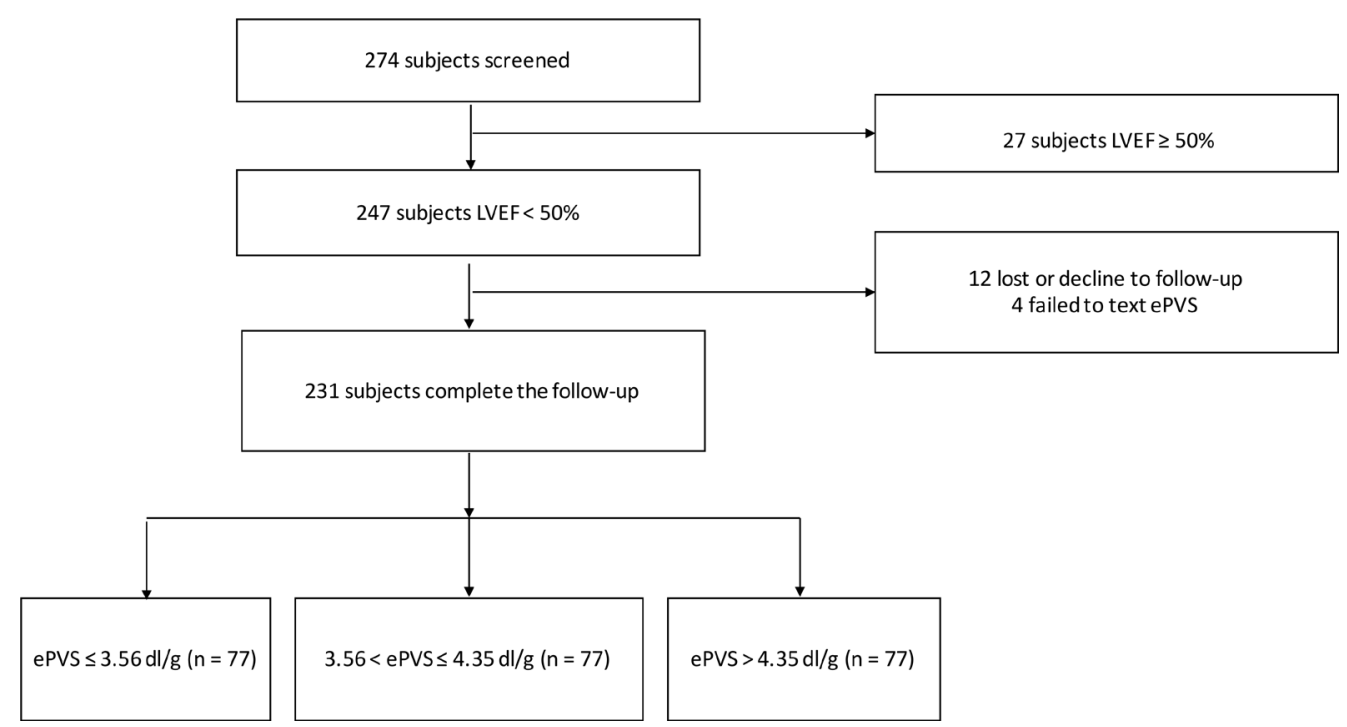

Figure 1 Flowchart of enrollment and follow-up of study cohort. ePVS, estimated plasma volume status; LVEF, left ventricular ejection fraction.

the predictive accuracy for each model. Receiver operating characteristic (ROC) curve analyses were performed to calculate areas under the curve (AUCs). Survival probabilities were estimated using the Kaplan-Meier method and were plotted as survival curves, which were compared using the log-rank test. Subgroup analyses were performed using univariable Cox regression analyses for primary outcome: reduced and mid-range LVEF. As the patient may die before rehospitalization, the assumption that censored observations have the same rehospitalization hazard as those at risk is not fulfilled; therefore, we performed competingrisk survival regression analysis for rehospitalization. The interaction between ePVS and anemia for primary outcome was tested using two-way analysis of variance. A p value of $<0.05$ (two-tailed) was considered significant. Statistical analyses and graphing were performed using the IBM SPSS Statistics V.21.0, STATA V.13.1 and GraphPad Prism V.5.0 (GraphPad Software, Cary, North Carolina, USA).

\section{RESULTS}

\section{Baseline characteristic}

Overall, 274 patients were included. Of these, 27 had an EF of $\geq 50 \%$, and 12 were lost to follow-up or declined to continue. The vital status could not be assessed in four patients. The remaining 231 patients comprised the study group. The cohort was respectively categorized according to ePVS tertiles: lower tertile-ePVS $\leq 3.56 \mathrm{dL} / \mathrm{g}$, middle tertile-3.56<ePVS $\leq 4.35 \mathrm{dL} / \mathrm{g}$, and upper tertileePVS $>4.35 \mathrm{dL} / \mathrm{g}$. A flowchart for the inclusion and follow-up algorithms is shown in figure 1 . After a median follow-up period of 35.6 months, 138 patients (59.7\%) experienced the primary outcome, including 60 who died (26\%) and 122 who were rehospitalized for HF (52.8\%). Characteristics of the study population are shown in online supplemental table S1.

\section{Factors associated with estimated PV at baseline}

The average age in the study was $61.98 \pm 9.11$ years and $62.3 \%$ were male (table 1). Patients with higher ePVS were more likely to be female, older and to have lower weights and BMIs. In addition, the uric acid, creatinine, hemoglobin, and hematocrit concentrations were significantly lower, while New York Heart Association (NYHA) classes were higher. The NT-proBNP was significantly different between the three groups $(1602,2726$, and $3035 \mathrm{pg} / \mathrm{mL}$ for the first, second, and third ePVS tertiles, respectively; $\mathrm{p}=0.001$ ). There were no significant differences between the three groups with respect to medications use. Most medical history features and laboratory values were comparable among the groups (table 1 ).

\section{ePVS and outcomes}

In the univariable Cox regression analyses, higher ePVS values were significantly associated with increased rates of the primary outcome (HR 1.659, 95\% CI 1.358 to 2.027; $\mathrm{p}<0.001$ ) (online supplemental table S3). After adjusting for MAGGIC score and $\operatorname{logBNP}$, ePVS remained an independent predictor of primary outcome (adjusted HR 1.567 , $95 \% \mathrm{CI} 1.267$ to $1.936 ; \mathrm{p}<0.001$ ) (table 2 ). To test the statistical concordance, we used the Harrel C-index to quantify the predictive accuracy of the multivariate models (table 2). For a multivariate Cox model with ePVS as independent variable, the $\mathrm{C}$-index was 0.618 . When adding the MAGGIC score, this value increased to 0.646 . However, the C-index rose to 0.659 when adding $\operatorname{logBNP}$ to variables. Finally, when both the MAGGIC score and $\operatorname{logBNP}$ were added to the model, the $\mathrm{C}$-index rose to 0.662 , and the concordance of the model was higher than the original model that included only ePVS. Moreover, when taking competing risks into account, ePVS remained a predictor of HF rehospitalization (online supplemental figure S5).

No significant correlation was found between ePVS and LVEF (Pearson correlation $=-0.008, p=0.903$ ), while weak correlations were found among $\log B N P$, ePVS and $\log B N P$, and LEVF (online supplemental table S2). In the multivariable Cox regression analysis for primary outcome, the variations in individual hemoglobin and hematocrit values were not retained because of the expected correlations with ePVS 
Table 1 Overall patient characteristics according to ePVS tertiles

\begin{tabular}{|c|c|c|c|c|c|}
\hline Clinical characteristics & $\begin{array}{l}\text { Total } \\
(\mathrm{N}=231)\end{array}$ & $\begin{array}{l}\text { First } \\
(e P V S \leq 3.56 \mathrm{dL} / g) \\
(n=77)\end{array}$ & $\begin{array}{l}\text { Second } \\
(3.56<e P V S \leq 4.35 \mathrm{dL} / \mathrm{g}) \\
(\mathrm{n}=77)\end{array}$ & $\begin{array}{l}\text { Third } \\
(e P V S>4.35 \mathrm{dL} / \mathrm{g}) \\
(\mathrm{n}=77)\end{array}$ & $P$ value \\
\hline \multicolumn{6}{|l|}{ Clinical variables } \\
\hline Age (years) & $61.98 \pm 9.11$ & $59.30 \pm 8.74$ & $63.58 \pm 8.86$ & $63.04 \pm 9.21$ & 0.006 \\
\hline Male (\%) & $62.3, n=144$ & $90.9, n=70$ & $61.0, n=47$ & $35.1, n=27$ & $<0.001$ \\
\hline Smoking (\%) & $52.4, n=121$ & $80.5, n=62$ & $49.4, n=38$ & $27.3, n=21$ & $<0.001$ \\
\hline Alcohol consumption (\%) & $41.1, n=95$ & $55.8, n=43$ & $39.0, n=30$ & $28.6, n=22$ & 0.002 \\
\hline \multicolumn{6}{|l|}{ Medical history (\%) } \\
\hline Hypertension & $30.3, n=70$ & $33.8, n=26$ & $32.5, n=25$ & $24.7, n=19$ & 0.414 \\
\hline Coronary artery disease & $36.8, n=85$ & $46.8, n=36$ & $36.4, n=28$ & $27.3, n=21$ & 0.043 \\
\hline Diabetes mellitus & $8.7, n=20$ & $6.5, n=5$ & $10.4, n=8$ & $9.1, n=7$ & 0.682 \\
\hline Atrial fibrillation & $7.8, n=18$ & $9.1, n=7$ & $9.1, n=7$ & $5.2, n=4$ & 0.581 \\
\hline \multicolumn{6}{|c|}{ Symptoms and physical examination } \\
\hline \multicolumn{6}{|l|}{ NYHA functional class (\%) } \\
\hline 1 & 18.3 & 26.0 & 18.2 & 10.5 & 0.172 \\
\hline II & 57.0 & 53.2 & 57.1 & 60.5 & \\
\hline III & 24.8 & 20.8 & 24.7 & 28.9 & \\
\hline Lung rales (\%) & $16.0 \%, n=37$ & $18.2 \%, n=14$ & $7.8 \%, n=6$ & $22.1 \%, n=17$ & 0.113 \\
\hline Third heart sound (\%) & $27.7 \%, n=64$ & $28.6 \%, n=22$ & $24.7 \%, n=19$ & $29.9 \%, n=23$ & 0.349 \\
\hline Peripheral edema (\%) & $20.8 \%, n=48$ & $16.9 \%, n=13$ & $24.7 \%, n=19$ & $20.8 \%, n=16$ & 0.238 \\
\hline Systolic BP (mm Hg) & $121.41 \pm 21.12$ & $122.42 \pm 21.23$ & $125.06 \pm 20.73$ & $116.74 \pm 20.80$ & 0.044 \\
\hline Diastolic BP (mm Hg) & $76.11 \pm 11.34$ & $78.06 \pm 10.82$ & $78.01 \pm 11.73$ & $72.25 \pm 10.58$ & 0.001 \\
\hline Heart rate (beats/min) & $77.79 \pm 16.31$ & $79.68 \pm 15.53$ & $76.13 \pm 16.11$ & $77.56 \pm 17.25$ & 0.400 \\
\hline Weight (kg) & $59.81 \pm 11.55$ & $65.74 \pm 11.96$ & $58.96 \pm 10.72$ & $54.73 \pm 9.11$ & $<0.001$ \\
\hline $\mathrm{BMI}\left(\mathrm{kg} / \mathrm{m}^{2}\right)$ & $23.06 \pm 3.75$ & $24.30 \pm 4.06$ & $23.01 \pm 3.50$ & $21.86 \pm 3.30$ & $<0.001$ \\
\hline MAGGIC score & $17.35 \pm 5.36$ & $15.78 \pm 4.74$ & $18.24 \pm 5.43$ & $18.04 \pm 5.58$ & 0.007 \\
\hline NT-proBNP (pg/mL) & $2454 \pm 171$ & $1602 \pm 193$ & $2726 \pm 338$ & $3035 \pm 313$ & 0.001 \\
\hline ePVS & $3.98 \pm 0.90$ & $3.05 \pm 0.46$ & $3.92 \pm 0.21$ & $4.97 \pm 0.54$ & $<0.001$ \\
\hline \multicolumn{6}{|l|}{ Laboratory values } \\
\hline Sodium (mmol/L) & $140.23 \pm 2.87$ & $140.34 \pm 3.14$ & $140.14 \pm 2.31$ & $140.21 \pm 3.11$ & 0.909 \\
\hline Potassium (mmol/L) & $4.34 \pm 0.46$ & $4.31 \pm 0.50$ & $4.34 \pm 0.49$ & $4.37 \pm 0.40$ & 0.749 \\
\hline Albumin (g/dL) & $4.27 \pm 0.34$ & $4.33 \pm 0.35$ & $4.27 \pm 0.37$ & $4.21 \pm 0.29$ & 0.089 \\
\hline Uric acid (mg/dL) & $326.32 \pm 88.09$ & $355.79 \pm 76.99$ & $322.81 \pm 92.41$ & $300.35 \pm 86.27$ & $<0.001$ \\
\hline BUN (mg/dL) & $19.12 \pm 5.12$ & $18.81 \pm 5.51$ & $19.51 \pm 4.30$ & $19.06 \pm 5.46$ & 0.804 \\
\hline Creatinine (mg/dL) & $0.78 \pm 0.17$ & $0.81 \pm 0.18$ & $0.78 \pm 0.16$ & $0.74 \pm 0.17$ & 0.021 \\
\hline eGFR $\left(\mathrm{mL} / \mathrm{min} / 1.73 \mathrm{~m}^{2}\right)$ & $86.53 \pm 18.94$ & $90.37 \pm 18.40$ & $85.18 \pm 19.87$ & $84.03 \pm 18.13$ & 0.086 \\
\hline Hemoglobin $(g / L)$ & $146.5 \pm 19.7$ & $166.9 \pm 14.8$ & $145.7 \pm 05.9$ & $126.9 \pm 10.2$ & $<0.001$ \\
\hline Hematocrit (\%) & $43.33 \pm 6.23$ & $49.63 \pm 5.17$ & $43.01 \pm 2.24$ & $37.36 \pm 3.07$ & $<0.001$ \\
\hline \multicolumn{6}{|l|}{ Echocardiography } \\
\hline LVEF (\%) & $35.75 \pm 7.65$ & $35.94 \pm 7.61$ & $35.11 \pm 7.59$ & $36.19 \pm 7.81$ & 0.658 \\
\hline $\operatorname{LVEDD}(\mathrm{mm})$ & $69.06 \pm 8.79$ & $69.13 \pm 8.76$ & $70.27 \pm 8.78$ & $67.79 \pm 8.79$ & 0.216 \\
\hline $\operatorname{LVESD}(\mathrm{mm})$ & $56.49 \pm 9.06$ & $56.53 \pm 8.92$ & $57.79 \pm 8.78$ & $55.16 \pm 9.40$ & 0.196 \\
\hline E wave $(\mathrm{m} / \mathrm{s})$ & $0.76 \pm 0.05$ & $0.75 \pm 0.04$ & $0.77 \pm 0.05$ & $0.75 \pm 0.04$ & 0.940 \\
\hline A wave $(\mathrm{m} / \mathrm{s})$ & $0.73 \pm 0.02$ & $0.76 \pm 0.04$ & $0.78 \pm 0.04$ & $0.67 \pm 0.04$ & 0.112 \\
\hline E:A ratio & $1.25 \pm 0.07$ & $1.21 \pm 0.12$ & $1.14 \pm 0.11$ & $1.39 \pm 0.13$ & 0.339 \\
\hline \multicolumn{6}{|l|}{ Medications } \\
\hline ACEI/ARB (\%) & $88.7 \%, n=205$ & $92.2 \%, n=71$ & $90.9 \%, n=70$ & $83.1 \%, n=64$ & 0.105 \\
\hline Beta blocker (\%) & $81.4 \%, n=188$ & $87.0 \%, n=67$ & $77.9 \%, n=60$ & $79.2 \%, n=61$ & 0.293 \\
\hline Aldosterone antagonist (\%) & $77.5 \%, n=179$ & $79.2 \%, n=61$ & $76.6 \%, n=59$ & $76.6 \%, n=59$ & 0.905 \\
\hline Diuretics (\%) & $56.3 \%, n=130$ & $54.5 \%, n=42$ & $55.8 \%, n=43$ & $58.4 \%, n=45$ & 0.884 \\
\hline Digitalis (\%) & $21.6 \%, n=50$ & $18.2 \%, n=14$ & $27.3 \%, n=21$ & $19.5 \%, n=15$ & 0.334 \\
\hline Coenzyme Q10 (\%) & $12.1 \%, n=28$ & $12.9 \%, n=10$ & $7.8 \%, n=6$ & $15.6 \%, n=12$ & 0.410 \\
\hline
\end{tabular}

Values are mean \pm SD, $n(\%)$ or median (25th-75th percentile).

ACEI, ACE inhibitor; ARB, angiotensin receptor blocker; BMI, body mass index; BNP, pro-brain natriuretic peptide; BP, blood pressure; BUN, blood urea nitrogen; eGFR, estimated glomerular filtration rate; ePVS, estimated plasma volume status; LVEF, left ventricular ejection fraction; LVEDD, left ventricular end-diastolic diameter; LVESD, left ventricular end-systolic diameter; MAGGIC, Meta-analysis Global Group in Chronic Heart Failure; NYHA, New York Heart Association; NT-proBNP, N-terminal pro-brain natriuretic peptide.

(Pearson correlation $=-0.954$ for hemoglobin variation, Pearson correlation $=-0.923$ for hematocrit variation) and with a less significant $\mathrm{p}$ value (table 3 ). ePVS was a better predictor of primary outcome than hemoglobin and hematocrit. Of note, in the subgroup analyses of both reduced and mid-range LVEF, higher ePVS values were also associated 


\begin{tabular}{|c|c|c|c|}
\hline Model & HR $(95 \% \mathrm{Cl})$ for ePVS & $P$ value & C-index \\
\hline ePVS crude HR & 1.659 (1.358 to 2.027$)$ & $<0.001$ & 0.618 \\
\hline $\begin{array}{l}\text { Model } 1 \\
\text { ePVS+age+gender }\end{array}$ & 1.659 (1.358 to 2.027$)$ & $<0.001$ & 0.616 \\
\hline $\begin{array}{l}\text { Model } 2 \text { ePVS+MAGGIC } \\
\text { score }\end{array}$ & 1.637 (1.333 to 2.011$)$ & $<0.001$ & 0.646 \\
\hline Model 3 ePVS+logBNP & 1.552 (1.258 to 1.914$)$ & $<0.001$ & 0.659 \\
\hline $\begin{array}{l}\text { Model } 4 \text { ePVS+MAGGIC } \\
\text { score+logBNP }\end{array}$ & 1.567 (1.267 to 1.936$)$ & $<0.001$ & 0.662 \\
\hline
\end{tabular}

Multivariable analysis results are reported for model 1, which included variables ePVS, age and gender. Multivariable model 2 included variables ePVS and MAGGIC score. Multivariable model 3 included variables ePVS and logBNP. Multivariable model 4 included variables ePVS, MAGGIC score and $\log B N P$.

BNP, pro-brain natriuretic peptide; ePVS, estimated plasma volume status; LVEF, left ventricular ejection fraction; MAGGIC score, Meta-analysis Global Group in Chronic Heart Failure; logBNP, normalized BNP with logarithmic transformation.

with increased rates of the primary outcome (online supplemental table S4). Furthermore, as shown in online supplemental table S6, there was no interactive effect of ePVS and anemia on primary outcome ( $\mathrm{p}$ interaction $=0.275$ ).

ePVS $(\mathrm{AUC}=0.645)$ and $\log \mathrm{BNP}(\mathrm{AUC}=0.692)$ were both good predictors of primary outcome (figure $2 \mathrm{~A}$ ). Similarly, for all-cause death, ePVS (AUC $=0.638)$ and $\log B N P$ $(\mathrm{AUC}=0.711$ ) were also good predictors (figure 2B). Analysis for HF rehospitalization, ePVS (AUC=0.647) and $\operatorname{logBNP}$ (AUC=0.648) were also good predictors (figure 2C). To further explore the importance of measuring ePVS at the time of clinical follow-up, Kaplan-Meier survival analysis was performed with ePVS tertiles. KaplanMeier estimates of the primary outcome stratified by ePVS tertiles showed that patients with CHF with higher baseline ePVS were predisposed to a greater risk of primary outcome (log-rank test: $p=0.006)$. The curve is shown in figure 3A. A similar trend was observed between ePVS and all-cause death (log-rank test: $p=0.014$ ) (figure 3B). Analysis of HF rehospitalization was also significant for log-rank test $(\mathrm{p}=0.001)$ (figure 3C).

\section{DISCUSSION}

Using a long follow-up prospective CHF cohort, we found that ePVS derived from hemoglobin and hematocrit
Table 3 Multivariable Cox regression analysis for primary outcome

\begin{tabular}{lll}
\hline $\begin{array}{l}\text { Variables retained by the } \\
\text { model }\end{array}$ & HR $(95 \% \mathrm{Cl})$ & P value \\
\hline ePVS & $1.554(1.263$ to 1.914$)$ & $<0.001$ \\
logBNP & $1.747(1.140$ to 2.676$)$ & 0.010 \\
LVEF & $0.966(0.945$ to 0.987$)$ & 0.002 \\
\hline
\end{tabular}

ePVS was retained in the Cox regression analysis model (HR: $1.554,95 \% \mathrm{Cl}$ 1.263 to $1.914 ; p<0.001)$. Variables: age, gender, systolic BP, MAGGIC score, $\operatorname{logBNP}$, ePVS, hemoglobin, hematocrit and LVEF.

$B N P$, pro-brain natriuretic peptide; BP, blood pressure; ePVS, estimated plasma volume status; logBNP, normalized BNP with logarithmic transformation.LVEF, left ventricular ejection fraction; MAGGIC score, Meta-analysis Global Group in Chronic Heart Failure;

predicted HF hospitalization or mortality events independently of major variables. This formula includes both hemoglobin and hematocrit, which may be relevant in patients with HF with cardiorenal anemia syndrome. ${ }^{27}$ Although both hematocrit and hemoglobin were associated with outcomes under univariate analyses, ePVS was a better predictor than hemoglobin and hematocrit in the multivariable analysis due to the collinearity with ePVS and with a less significant $\mathrm{p}$ value.

Daily body weights reflect daily fluctuations in PV, but this is insufficiently informative over a longer period and only accurate when the patient's dry weight is known. ${ }^{13} \mathrm{In}$ addition, body weight loss, which was found to be related to worse outcomes, may rather be related to cachexia as opposed to decongestion and therefore may be misleading for use in monitoring congestive status. ${ }^{13}$ In our study, there was a trend that patients with higher ePVS had severe clinical signs and symptoms of congestion. NYHA classes were higher when patients had higher ePVS, although there was no significant difference. For lung rales and edema, the trend was not consistent. Many factors can affect the symptoms and signs of patients with CHF; congestion is one of the factors. ${ }^{8}$ Symptoms and signs can only be used to quantify PV when congestion is apparent. ${ }^{8}$ The correlation between vascular volume and clinical symptoms and signs is complex and requires further exploration.

An analysis reported that ePVS was associated with invasively measured left ventricular end-diastolic pressure, ${ }^{28}$ whereas ePVS was preferably associated with leftsided hemodynamic markers of congestion. ${ }^{28}$ These results indicate that $\mathrm{ePVS}$ is a congestion variable rather than a
A

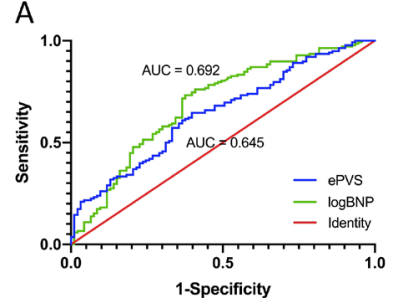

B

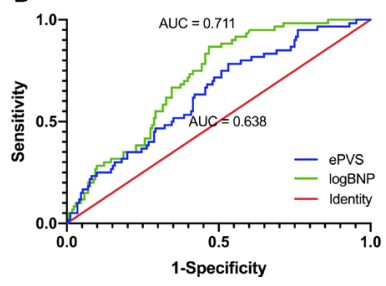

C

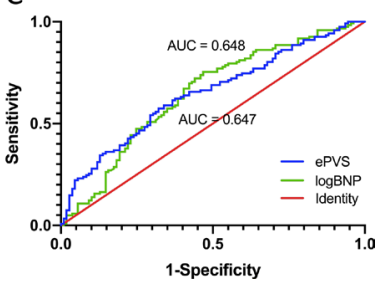

Figure 2 ROC curves related to NT-proBNP and ePVS. ROC curves for logBNP (AUC $=0.692)$ and ePVS (AUC $=0.645)$ measurements. Relation of ePVS and logBNP to primary outcome (A). ROC curves related to logBNP (AUC $=0.711$ ) and ePVS (AUC $=0.638)$ for all-cause death (B). ROC curves related to $\log B N P(A U C=0.648)$ and ePVS (AUC $=0.647$ ) for rehospitalization (C). AUC, area under the curve; BNP, brain natriuretic peptide; ePVS, estimated plasma volume status; NT-proBNP, N-terminal pro-brain natriuretic peptide; ROC, receiver operating characteristic. 
A

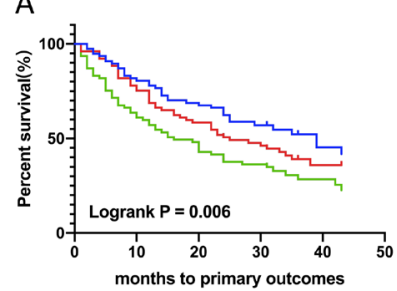

B

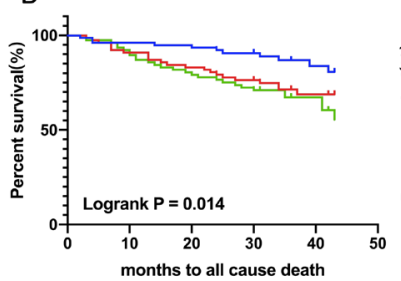

C

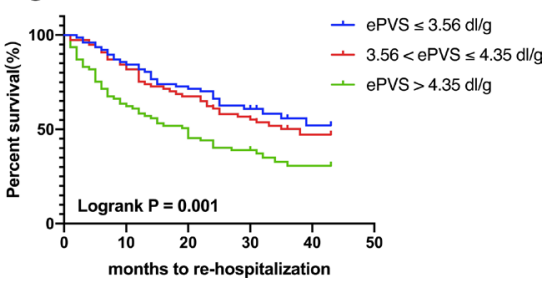

Figure 3 Kaplan-Meier survival curves according to ePVS tertiles. Kaplan-Meier curves for patients were divided into tertiles of ePVS from baseline to the end of the follow-up. Relation of ePVS to primary outcome (A), all-cause death (B) and rehospitalization (C). ePVS, estimated plasma volume status.

comorbidity variable. In addition to traditional routine clinical assessments, this ePVS method allows physicians to assess a patient's congestive status and to predict future prognosis.

PV estimation is important in the management of patients with HF to tailor diuretic doses to the needs of the individual patient, but is often not achieved due to the unreliability of clinical signs and symptoms. ${ }^{29}$ The ePVS measurements may help physicians estimate PV and adjust guideline-based medications. Patients with HF are not always able to undergo outpatient clinic treatment, medication, or follow-up in centers with various biomarkers laboratory tests. ${ }^{30}$ In these situations, hemoglobin and hematocrit to estimate PV is a low-cost, easily measurable alternative method available in clinical practice.

Our study had some limitations. First, our study was an observational cohort, with the usual limitations of such protocols. Second, although we found that the ePVS showed a consistent trend in predicting the outcomes at the end of the follow-up period, we believe that we have not been able to obtain the variation of ePVS over time. Using more time series may be more clinically significant and predictive to replace a single test with a baseline test. Third, we included only two centers. The number of patients in the cohort was relatively small. Although we obtained longterm independent forecast values, the overall power was not high. Prospective cohort studies with larger sample sizes in the future can provide more reliable evidence. Finally, the therapy associated results presented here are largely hypothetical. Therefore, prospective studies are required to fully evaluate the therapy-associated potential of ePVS. Despite these limitations, we believe that our research has potential clinical implications. Our study further strengthens the evidence for an important prognostic value of ePVS in patients with stable systolic CHF.

\section{CONCLUSION}

In summary, ePVS calculated simply from hemoglobin and hematocrit independently provided a predictive value for long-term HF hospitalization or mortality outcome in patients with stable systolic CHF.

Contributors YL: methodology, writing and original draft preparation; $Y X$ : supervision and investigation; JL: data curation and investigation; $X W$ : validation and investigation; LW: data curation; JL: investigation; LB: supervision, writing, reviewing and editing the manuscript; AM: conceptualization, writing, reviewing and editing.
Funding This work was funded by the Ministry of Science and Technology of the People's Republic of China grant (number 2017YFC1308302) and Ministry of Finance of the People's Republic of China (number 2012GS610101).

Competing interests None declared.

Patient consent for publication Not required.

Ethics approval The study was authorized by the ethics committee of the First Affiliated Hospital of Xi'an Jiaotong University (approved number of ethic committee: 2013-133) and was conducted in accordance with the Declaration of the Helsinki. All participants signed informed consent forms.

Provenance and peer review Not commissioned; externally peer reviewed. Data availability statement Data are available upon reasonable request. The deidentified participant data will not be shared.

Supplemental material This content has been supplied by the author(s). It has not been vetted by BMJ Publishing Group Limited (BMJ) and may not have been peer-reviewed. Any opinions or recommendations discussed are solely those of the author(s) and are not endorsed by BMJ. BMJ disclaims all liability and responsibility arising from any reliance placed on the content. Where the content includes any translated material, BMJ does not warrant the accuracy and reliability of the translations (including but not limited to local regulations, clinical guidelines, terminology, drug names and drug dosages), and is not responsible for any error and/or omissions arising from translation and adaptation or otherwise.

Open access This is an open access article distributed in accordance with the Creative Commons Attribution Non Commercial (CC BY-NC 4.0) license, which permits others to distribute, remix, adapt, build upon this work noncommercially, and license their derivative works on different terms, provided the original work is properly cited, an indication of whether changes were made, and the use is non-commercial. See: http://creativecommons.org/ licenses/by-nc/4.0/.

\section{ORCID iDs}

Yuyao Lin http://orcid.org/0000-0002-3683-1960

Aiqun Ma http://orcid.org/0000-0002-4622-5227

\section{REFERENCES}

1 Jessup M, Brozena S. Heart failure. N Engl J Med 2003;348:2007-18.

2 Anand IS, Ferrari R, Kalra GS, et al. Edema of cardiac origin. studies of body water and sodium, renal function, hemodynamic indexes, and plasma hormones in untreated congestive cardiac failure. Circulation 1989;80:299-305.

3 Dupont M, Mullens W, Tang WHW. Impact of systemic venous congestion in heart failure. Curr Heart Fail Rep 2011;8:233-41.

4 Kalra PR, Anagnostopoulos C, Bolger AP, et al. The regulation and measurement of plasma volume in heart failure. J Am Coll Cardiol 2002;39:1901-8.

5 Drazner MH, Rame JE, Stevenson LW, et al. Prognostic importance of elevated jugular venous pressure and a third heart sound in patients with heart failure. N Engl J Med 2001;345:574-81.

6 Damman K, Voors AA, Hillege HL, et al. Congestion in chronic systolic heart failure is related to renal dysfunction and increased mortality. Eur J Heart Fail 2010;12:974-82.

7 Miller WL, Mullan BP. Understanding the heterogeneity in volume overload and fluid distribution in decompensated heart failure is key to optimal volume management: role for blood volume quantitation. JACC Heart Fail 2014:2:298-305 
8 Stevenson LW, Perloff JK. The limited reliability of physical signs for estimating hemodynamics in chronic heart failure. JAMA 1989;261:884-8.

9 Girerd N, Seronde M-F, Coiro S, et al. Integrative Assessment of Congestion in Heart Failure Throughout the Patient Journey. JACC Heart Fail 2018:6:273-85.

10 Lang RM, Bierig M, Devereux RB, et al. Recommendations for chamber quantification. Eur J Echocardiogr 2006;7:79-108.

11 Ponikowski P, Voors AA, Anker SD, et al. ESC Guidelines for the diagnosis and treatment of acute and chronic heart failure: The Task Force for the diagnosis and treatment of acute and chronic heart failure of the European Society of Cardiology (ESC)Developed with the special contribution of the Heart Failure Association (HFA) of the ESC. Eur Heart J 2016;2016:2129-200.

12 Recommended methods for measurement of red-cell and plasma volume: International Committee for standardization in haematology. J Nucl Med 1980;21:793-800.

13 Chaudhry SI, Wang Y, Concato J, et al. Patterns of weight change preceding hospitalization for heart failure. Circulation 2007;116:1549-54.

14 Duarte K, Monnez J-M, Albuisson E, et al. Prognostic value of estimated plasma volume in heart failure. JACC Heart Fail 2015;3:886-93.

15 Ling HZ, Flint J, Damgaard M, et al. Calculated plasma volume status and prognosis in chronic heart failure. Eur J Heart Fail 2015;17:35-43.

16 Kobayashi M, Girerd N, Duarte K, et al. Prognostic impact of plasma volume estimated from hemoglobin and hematocrit in heart failure with preserved ejection fraction. Clin Res Cardiol 2020;109:1392-401.

17 Huang C-Y, Lin T-T, Wu Y-F, et al. Long-Term prognostic value of estimated plasma volume in heart failure with preserved ejection fraction. Sci Rep 2019;9:14369.

18 Massari F, Scicchitano P, lacoviello M, et al. Multiparametric approach to congestion for predicting long-term survival in heart failure. J Cardiol 2020;75:47-52.

19 Riegel B, Moser DK, Anker SD, et al. State of the science: promoting self-care in persons with heart failure: a scientific statement from the American Heart Association. Circulation 2009;120:1141-63.

20 McMurray JJV, Adamopoulos S, Anker SD, et al. ESC guidelines for the diagnosis and treatment of acute and chronic heart failure 2012: the task force for the diagnosis and treatment of acute and chronic heart failure 2012 of the European Society of cardiology. developed in collaboration with the heart failure association (HFA) of the ESC. Eur Heart J 2012;33:1787-847

21 Whelton PK, Carey RM, Aronow WS, et al. 2017 ACC/AHA/AAPA/ABC/ACPM/ AGS/APhA/ASH/ASPC/NMA/PCNA guideline for the prevention, detection, evaluation, and management of high blood pressure in adults: Executive summary: a report of the American College of Cardiology/American heart association Task force on clinical practice guidelines. J Am Soc Hypertens 2018;12:579 e571-9.

22 American Diabetes Association. Diagnosis and classification of diabetes mellitus. Diabetes Care 2004;27 Suppl 1:S5-10.

23 January CT, Wann LS, Alpert JS, et al. 2014 AHA/ACC/HRS guideline for the management of patients with atrial fibrillation: Executive summary: a report of the American College of Cardiology/American heart association Task force on practice guidelines and the heart rhythm Society. Circulation 2014;130:2071-104.

24 Garrow JS, Webster J. Quetelet's index (W/H2) as a measure of fatness. Int J Obes 1985;9:147-53.

25 Levey AS, Coresh J, Balk E, et al. National kidney Foundation practice guidelines for chronic kidney disease: evaluation, classification, and stratification. Ann Intern Med 2003;139:137-47.

26 Pocock SJ, Ariti CA, McMurray JJV, et al. Predicting survival in heart failure: a risk score based on 39372 patients from 30 studies. Eur Heart J 2013;34:1404-13.

27 Davenport A, Anker SD, Mebazaa A, et al. ADQI 7: the clinical management of the Cardio-Renal syndromes: work group statements from the 7th ADQI consensus conference. Nephrol Dial Transplant 2010;25:2077-89.

28 Kobayashi M, Huttin O, Donal E, et al. Association of estimated plasma volume status with hemodynamic and echocardiographic parameters. Clin Res Cardiol 2020;109:1060-9.

29 Damman K, Testani JM. The kidney in heart failure: an update. Eur Heart J 2015;36:1437-44.

30 Lainscak M, Blue L, Clark AL, et al. Self-Care management of heart failure: practical recommendations from the patient care Committee of the heart failure association of the European Society of cardiology. Eur J Heart Fail 2011;13:115-26. 